\title{
CARACTERIZAÇÃO DE COMITÊS DE ÉTICA EM PESQUISA DE UM MUNICÍPIO BAIANO
}

\section{CHARACTERIZATION OF ETHICS COMMITTEES IN RESEARCH OF A MUNICIPALITY OF BAHIA}

\section{CARACTERIZACIÓN DE COMITÉS DE ÉTICA EN INVESTIGACIÓN DE UN MUNICIPIO DE BAHÍA}

\author{
Rosinete Souza Barata ${ }^{1}$ \\ Karla Ferraz dos Anjos ${ }^{2}$ \\ Alciene Pereira da Silva ${ }^{3}$ \\ Julia Barbosa de Magalhães ${ }^{4}$ \\ Darci de Oliveira Santa Rosa
}

Como citar este artigo: Barata RS, Anjos KF, Silva AP, Magalhães JB, Rosa DOS. Caracterização de Comitês de Ética em Pesquisa de um município baiano. Rev baiana enferm. 2021;35:e45319.

Objetivo: caracterizar o perfil dos Comitês de Ética em Pesquisa de um município baiano. Método: estudo de abordagem quantitativa, com análise descritiva dos resultados, realizado em cinco Comitês de Ética em Pesquisa. A coleta de dados deu-se por questionário autoaplicado na Plataforma Google Forms. Resultados: evidenciou-se maioria de membros do gênero masculino, com curso de pós-graduação stricto sensu, ingresso por convite e capacitação prévia para atuarem nos Comitês. Foram implantados há mais de 8 anos; composição varia de 8 a 25 membros efetivos; predomina a utilização da página eletrônica no processo comunicativo. Os projetos avaliados são multidisciplinares, distribuídos para apreciação considerando a área de formação, perfil e expertise do membro. Conclusão: os Comitês de Ética em Pesquisa estudados atuavam há mais de oito anos, o que demonstra experiência consolidada em pesquisa. A recondução do mandato contribui para o aprofundamento das discussões, capacitação de novos membros e fortalecimento do sistema CEP/CONEP.

Descritores: Comitês de Ética em Pesquisa. Ética em Pesquisa. Revisão Ética. Humanos. Bioética.

Objective: to characterize the profile of the Research Ethics Committees of a municipality of Babia. Method: quantitative approach study, with descriptive analysis of the results, conducted in five Research Ethics Committees. Data collection was based on a self-administered questionnaire on the Google Forms Platform. Results: a majority of male members were evidenced, with stricto sensu graduate course, invitation admission and prior training to work in the Committees. They were implanted more than 8 years ago; composition ranges from 8 to 25 full members; the use of the website in the communicative process predominates. The projects evaluated are multidisciplinary, distributed for consideration considering the area of training, profile, and expertise of the member. Conclusion: the Research Ethics Committees studied had been active for more than eight years, which demonstrates consolidated experience in research. The renewal of the mandate contributes to the deepening of discussions, training of new members and strengthening of the system (CEP/CONEP in Portuguese).

Descriptors: Research Ethics Committees. Ethics in Research. Ethics Review. Human. Bioethics.

\footnotetext{
Enfermeira. Advogada. Mestra em Enfermagem e Saúde. Universidade Federal da Bahia. Salvador, Bahia, Brasil. rsouzabarata.enfa@gmail.com. https://orcid. org/0000-0002-9084-1625.

Enfermeira. Pós-doutora em Enfermagem e Saúde. Professora da União Metropolitana de Educação e Cultura. Salvador, Bahia, Brasil. https://orcid.org/0000-00025453-8303.

Enfermeira. Mestra em Meio Ambiente. Universidade Federal da Bahia. Salvador, Bahia, Brasil. https://orcid.org/0000-000 I-8047-5994.

4 Enfermeira. Pesquisadora independente. Salvador, Bahia, Brasil. https://orcid.org/0000-000 I-9294-8672.

Enferneira. Pós-doutora em Bioética. Docente do Programa de Pós-Graduação da Universidade Federal da Bahia. Salvador, Bahia, Brasil. https://orcid.org/0000$0002-5651-2916$
} 
Objetivo: caracterizar el perfil de los Comités de Ética en Investigación de un municipio de Babía. Método: estudio de aproximación cuantitativa, con análisis descriptivo de los resultados, realizado en cinco Comités de Ética en Investigación. La recolección de datos se basó en un cuestionario autoadministrado en Google Forms Platform. Resultados: se evidenció la mayoría de los miembros varones, con curso de posgrado stricto sensu, admisión por invitación y capacitación previa para trabajar en los Comités. Fueron implantados hace más de 8 años; la composición oscila entre 8 y 25 miembros de pleno derecho; predomina el uso del sitio web en el proceso comunicativo. Los proyectos evaluados son multidisciplinarios, distribuidos para su consideración considerando el área de capacitación, perfil y experiencia del miembro. Conclusión: los Comités de Ética en Investigación estudiados llevaban más de ocho años activos, lo que demuestra una experiencia consolidada en investigación. La renovación del mandato contribuye a la profundización de los debates, la capacitación de nuevos miembros y el fortalecimiento del sistema (CEP/CONEP en Portugués).

Descriptores: Comités de Ética en Investigación. Ética en la Investigación. Revisión Ética. Humano. Bioética.

\section{Introdução}

Os Comitês de Ética em Pesquisa (CEPs) são órgãos responsáveis pela apreciação de protocolos de pesquisas envolvendo seres humanos submetidos à Plataforma Brasil, oriundos de instituições públicas e particulares. Têm por finalidade a defesa e a proteção dos direitos dos participantes da pesquisa ${ }^{(1)}$. Desse modo, os CEPs são instâncias de consulta obrigatória para que pesquisas científicas que envolvam seres humanos possam ser iniciadas.

Nesse sentido, os CEPs contribuem para a qualidade das pesquisas e para a discussão da função social que elas exercem nas instituições onde são desenvolvidas e para a comunidade em geral. Consequentemente, valorizam e defendem os interesses do participante e do pesquisador, pois, uma vez aprovado, o protocolo de pesquisa será validado como uma proposta adequada do ponto de vista ético ${ }^{(2)}$. Por essa razão é necessário que os pesquisadores norteiem suas pesquisas de acordo com os requisitos éticos definidos nos documentos e normativas de pesquisas que envolvem seres humanos cujo principal enfoque relaciona-se ao respeito aos direitos humanos e à dignidade da pessoa humana ${ }^{(3)}$.

Outrossim, os CEPs realizam a revisão ética dos protocolos de pesquisa ao mesmo tempo que associam essa revisão à análise científica e mantém as informações conhecidas em caráter estritamente confidencial ${ }^{(4)}$. Na tomada de decisões, cada Comitê de Ética em Pesquisa deve ter total independência e priorizar a apreciação dos protocolos de relevância pública e de interesse estratégico da agenda de prioridades do Sistema Único de Saúde (SUS). Ademais, o CEP deve emitir parecer dos protocolos de pesquisa a ele submetidos nos prazos estabelecidos pela norma operacional específica ${ }^{(5)}$. Além da função de realizar a revisão ética dos projetos de pesquisa, os CEPs têm função educativa, consultiva e deliberativa $^{(2)}$.

A implantação de um CEP é feita sob forma de colegiado multidisciplinar e independente, para analisar e decidir acerca dos protocolos de pesquisas que envolvem seres humanos ${ }^{(6)}$. Por ser colegiado, cada CEP deve ser formado por, pelo menos, sete membros e registrado na Comissão Nacional de Ética em Pesquisa (CONEP), instância encarregada de analisar a documentação e solicitação desse registro. Além disso, o CEP deve comprovar que, no mínimo, 50\% de seus membros possuem experiência em pesquisa. Todavia, pode haver variação nessa composição, a depender da especificidade de cada instituição e das temáticas de pesquisa analisadas ${ }^{(7)}$.

$\mathrm{Na}$ distribuição dos membros de cada CEP, conforme orientação da CONEP, deve ser respeitado o princípio da proporcionalidade em relação à categoria profissional e à participação dos dois sexos. Nesse sentido, esses membros devem pertencer a diferentes áreas do conhecimento e categorias profissionais, para atender aos aspectos multidisciplinar e multiprofissional necessários à composição do CEP e deve haver, no mínimo, um representante dos usuários. 
Aliado a isso, para contemplar o perfil de multidisciplinariedade, poderão ser convidadas como membros efetivos pessoas de outras instituições e de áreas distintas do conhecimento e deve incluir estudiosos da Bioética ${ }^{(2)}$. Os aspectos de multidisciplinariedade e proporcionalidade devem ser descritos em formulário próprio, com as informações do nome completo de cada membro, sua atribuição no CEP, o gênero, a formação profissional, a área de conhecimento e a escolaridade atual ${ }^{(8)}$.

A duração do mandato do membro é de três anos, mas pode haver recondução por igual período. Compete a cada Comitê definir a forma de ingresso de membros no CEP, que pode ser, por exemplo, por meio de eleição, edital, indicação e convite. Uma vez definida a forma de ingresso dos membros, esta deve constar no seu regimento interno $(\mathrm{RI})^{(2)}$. Por outro lado, o ingresso do membro representante dos usuários no CEP é feito por indicação, de preferência, pelos Conselhos Municipais ou Estaduais de Saúde, por movimentos sociais e entidades representativas dos usuários ${ }^{(7)}$.

Estudar a forma de atuação do CEP é considerada prioridade pela Agenda Nacional de Prioridades de Pesquisa em Saúde (ANPPS) ${ }^{(9)}$, pois possibilita, entre outros aspectos, evidenciar a necessidade de ações de capacitação de seus membros para aprofundamento dos temas apreciados nos projetos de pesquisa. Além disso, os CEPs são órgãos de relevância pública no controle social da pesquisa ${ }^{(10)}$.

Conhecer esses comitês pode ajudar pesquisadores de todas as áreas do conhecimento a compreenderem o porquê da necessidade de desenvolverem suas pesquisas de acordo com aspectos que gerem segurança e proteção aos indivíduos ${ }^{(11)}$. Consequentemente, trazem benefício para a sociedade, como é o caso de pesquisas cujos resultados são utilizados na área da saúde. Diante disso, o objetivo deste estudo é caracterizar o perfil dos CEPs de um município baiano.

\section{Método}

Estudo de abordagem quantitativa e análise descritiva dos resultados. A coleta de dados deu-se por meio de questionário autoaplicado na Plataforma Google Forms, no período de 10 de setembro a 13 de outubro de 2020. Foram estudadas: variáveis sociodemográficas (sexo, cor, estado civil e escolaridade); variáveis estruturais do CEPs (tempo de existência, quantidade de membros, formação dos membros, duração dos mandatos, recondução dos membros, formas de relacionamento do CEP com o público e com outros CEPs); e variáveis que compõem o processo de distribuição, quantidade e áreas temáticas dos projetos apreciados no CEP.

O estudo envolveu cinco CEPs implantados em instituições públicas, localizados em um município baiano assim distribuídos: quatro CEPs implantados em instituições de ensino superior (IES), com predominância de CEP da área da saúde; um CEP implantado em hospital. Os participantes foram quatro coordenadores e um vice-coordenador. Adotou-se como critério de inclusão ser responsável pela apreciação de projetos de pesquisa e emissão de parecer. Os critérios de exclusão foram estar em licença médica, férias ou afastado por qualquer razão.

Frise-se que, do total de 39 CEPs existentes no município na data da coleta $^{(12)}$, selecionou-se, por conveniência, $10 \mathrm{CEPs}$, entretanto apenas 5 CEPs aceitaram responder às questões de caracterização. Para a caracterização do perfil e para que se evitasse a repetição e/ou a duplicidade de coleta de dados, foi inserido no questionário que apenas os coordenadores e/ou pessoa por ele indicada poderiam responder o questionário. Os contatos dos participantes foram disponibilizados pelos respectivos CEPs via e-mail. Foram observados os aspectos éticos relacionados à pesquisa que envolve seres humanos, conforme a Resolução n. 466/2012, do Conselho Nacional de Saúde, sobretudo no que diz respeito ao consentimento livre e esclarecido, sigilo e anonimato dos participantes. Nesse sentido, o Termo de Consentimento Livre e Esclarecido (TCLE) foi disponibilizado por meio de link inserido no questionário com possibilidade de download pelos participantes e assinatura virtual do TCLE à medida que aceitassem responder ao questionário. Os dados coletados foram organizados por CEP, em planilha 
Excel, e receberam codificação especifica para evitar a identificação dos participantes e seus respectivos CEPs.

Este estudo foi aprovado pelo CEP da Escola de Enfermagem da Universidade Federal da Bahia (EEUFBA), sob Parecer n. 4.255.337 e Certificado de Apresentação de Apreciação Ética (CAAE) 36248120.7.0000.5531 e faz parte do projeto de pesquisa de dissertação intitulada
"Problemas morais e/ou éticos vivenciados por membros de Comitês de Ética e Pesquisa”.

\section{Resultados}

Do total dos cinco participantes do estudo, quatro eram coordenadores de CEP e um vice-coordenador (CEP 5), conforme descrito no Quadro 1, a seguir.

Quadro 1 - Distribuição dos coordenadores e vice-coordenador por Comitê de Ética em Pesquisa quanto às características sociodemográficas

\begin{tabular}{|l|l|l|l|l|l|}
\hline CEP & Gênero & $\begin{array}{c}\text { Cor da pele } \\
\text { autodeclarada }\end{array}$ & \multicolumn{1}{|c|}{$\begin{array}{c}\text { Estado } \\
\text { civil }\end{array}$} & \multicolumn{1}{|c|}{ Maior escolaridade } & Religião \\
\hline A* & Masculino & Preta & Divorciado & Mestrado & $\begin{array}{l}\text { Cristianismo } \\
\text { (sem especificar) }\end{array}$ \\
\hline B & Feminino & Branca & Casada & Doutorado & Católica \\
\hline C & Masculino & Branca & Casado & Doutorado & Católica \\
\hline D & Masculino & Branca & Casado & $\begin{array}{l}\text { Pós-graduação em Psicologia } \\
\text { (sem especificar) }\end{array}$ & Católica \\
\hline E & Masculino & Preta & Casado & $\begin{array}{l}\text { Pós-graduação em } \\
\text { Antropologia } \\
\text { (sem especificar) }\end{array}$ & Candomblé \\
\hline
\end{tabular}

Fonte: Elaboração própria.

Legenda:

* Vice-Coordenador; CEP Comitê de Ética em Pesquisa.

Em relação aos cursos de graduação e categoria profissional dos coordenadores dos CEPs, verificou-se a mesma proporção de profissionais nas áreas de Saúde e Humanas. Desse modo, quanto aos cursos de formação, foram citados: odontologia, farmácia, administração, psicologia e filosofia. Já as categorias profissionais dos participantes foram: dentista, farmacêutico, administrador, psicólogo e antropólogo. Não houve duplicidade de curso de graduação e/ou categoria profissional.

Os participantes expressaram atuar no CEP da instituição na qual trabalham com tempo de atuação variando de 1,5 a 10 anos. Todos relataram ter recebido treinamento inicial para ser membro e capacitação continuada, que pode ocorrer a cada trinta dias e mesmo entre um e dois anos. A maioria (3) dos membros relatou inexistir incentivos para atuarem no CEP.

No Quadro 2, sintetiza-se as informações sobre o tempo de implantação (existência) dos CEPs do estudo, quantitativo de membros efetivos, forma de ingresso no respectivo CEP, forma de comunicação utilizada pelos CEPs para relacionamento com o público/pesquisadores, com outros CEPs e o CONEP e alteração nas formas e relacionamento em decorrência da Covid-19. Frise-se que, em decorrência da pandemia provocada pela Covid-19, a maioria descreveu que o relacionamento presencial foi suspenso e substituído por contatos telefônicos e videoconferências.

Quadro 2 - Variáveis estruturais dos Comitês de Ética em Pesquisa

\begin{tabular}{|c|c|c|c|c|c|}
\hline \multirow{2}{*}{ Variáveis } & \multicolumn{5}{|c|}{ Comitês de Ética em Pesquisa } \\
\hline & $\mathbf{A}$ & B & $\mathbf{C}$ & $\mathbf{D}$ & $\mathbf{E}$ \\
\hline $\begin{array}{l}\text { Tempo de } \\
\text { existência (anos) }\end{array}$ & Não respondido & 8 & 16 & $\begin{array}{l}\text { Não soube } \\
\text { informar }\end{array}$ & 15 \\
\hline
\end{tabular}


Quadro 2 - Variáveis estruturais dos Comitês de Ética em Pesquisa (conclusão)

\begin{tabular}{|c|c|c|c|c|c|}
\hline \multirow{2}{*}{ Variáveis } & \multicolumn{5}{|c|}{ Comitês de Ética em Pesquisa } \\
\hline & $\mathbf{A}$ & B & C & $\mathbf{D}$ & $\mathbf{E}$ \\
\hline $\begin{array}{l}\text { Quantidade } \\
\text { de membros } \\
\text { efetivos }\end{array}$ & 25 & 8 & 8 & 9 & 9 \\
\hline $\begin{array}{l}\text { Forma de } \\
\text { ingresso do } \\
\text { representante } \\
\text { de usuário }\end{array}$ & $\begin{array}{l}\text { Indicação de } \\
\text { uma instituição } \\
\text { /associação da } \\
\text { comunidade } \\
\text { civil }\end{array}$ & Convite & Não informado & $\begin{array}{l}\text { Indicação } \\
\text { de uma } \\
\text { instituição } \\
\text { /associação da } \\
\text { comunidade } \\
\text { civil }\end{array}$ & $\begin{array}{l}\text { Indicação } \\
\text { (sem } \\
\text { especificar) }\end{array}$ \\
\hline $\begin{array}{l}\text { Forma de } \\
\text { ingresso } \\
\text { dos demais } \\
\text { membros } \\
\text { efetivos }\end{array}$ & Eleição & Convite & Convite & Convite & Edital \\
\hline $\begin{array}{l}\text { Duração de } \\
\text { mandato dos } \\
\text { membros } \\
\text { (anos) }\end{array}$ & 3 & 3 & 3 & 2 & 3 \\
\hline $\begin{array}{l}\text { Recondução de } \\
\text { membros }\end{array}$ & Sim & $\operatorname{Sim}$ & $\operatorname{Sim}$ & Sim & $\operatorname{Sim}$ \\
\hline $\begin{array}{l}\text { Formas de } \\
\text { relacionamento } \\
\text { do CEP com o } \\
\text { público e/ou } \\
\text { pesquisadores }\end{array}$ & $\begin{array}{l}\text { Página } \\
\text { eletrônica; } \\
\text { Fale conoso; } \\
\text { Presencialmente }\end{array}$ & $\begin{array}{l}\text { Página } \\
\text { eletrônica }\end{array}$ & $\begin{array}{l}\text { Página } \\
\text { eletrônica; } \\
\text { Presencial; } \\
\text { e-mail; } \\
\text { telefone; } \\
\text { Página intranet }\end{array}$ & $\begin{array}{l}\text { Página } \\
\text { eletrônica }\end{array}$ & $\begin{array}{l}\text { Página } \\
\text { eletrônica }\end{array}$ \\
\hline $\begin{array}{l}\text { Formas de } \\
\text { relacionamento } \\
\text { do CEP com } \\
\text { outros CEPs e } \\
\text { com a CONEP }\end{array}$ & $\begin{array}{l}\text { Página } \\
\text { eletrônica; } \\
\text { Fale conoso; } \\
\text { Presencialmente }\end{array}$ & $\begin{array}{l}\text { Não } \\
\text { respondido }\end{array}$ & $\begin{array}{l}\text { Fale conoso; } \\
\text { Plataforma } \\
\text { Brasil; } \\
\text { WhatsApp. }\end{array}$ & $\begin{array}{l}\text { Página } \\
\text { eletrônica; } \\
\text { Grupo de } \\
\text { WhatsApp }\end{array}$ & $\begin{array}{l}\text { Página } \\
\text { eletrônica; } \\
\text { Fale conosco }\end{array}$ \\
\hline $\begin{array}{l}\text { Alteração nas } \\
\text { formas de } \\
\text { relacionamento } \\
\text { em decorrência } \\
\text { da Covid-19 }\end{array}$ & Sim & $\operatorname{Sim}$ & $\operatorname{Sim}$ & Não & Não \\
\hline
\end{tabular}

Fonte: Elaboração própria.

Legenda: CEP Comité de Ética em Pesquisa; CONEP Comissão Nacional de Ética em Pesquisa.

O processo de distribuição dos projetos de pesquisa nos CEPs ocorre de formas distintas inclusive para o mesmo CEP. As áreas temáticas dos projetos analisados com mais frequência são novos fármacos. Somente um CEP referiu a participação do ad hoc. Há CEPs com mais de uma forma de encontro para deliberar sobre os protocolos de pesquisa (Tabela 1).

Tabela 1 - Processo de distribuição, quantidade e áreas temáticas dos projetos apreciados, participação de ad hoc. Bahia, Brasil, 2020. ( $\mathrm{N}=5)$

\begin{tabular}{|c|c|c|c|c|}
\multicolumn{5}{c}{ Comitê de Ética em Pesquisa } \\
\hline A & B & C & D & E \\
\hline
\end{tabular}

\section{Processo de distribuição dos projetos}

Com base na área de formação do membro

Com base no perfil do membro

$\begin{array}{ll}1 & 1 \\ 1 & -\end{array}$


Tabela 1 - Processo de distribuição, quantidade e áreas temáticas dos projetos apreciados, participação de ad hoc. Bahia, Brasil, 2020. ( $\mathrm{N}=5)$

(conclusão)

\begin{tabular}{|c|c|c|c|c|c|}
\hline \multirow{2}{*}{ Variáveis } & \multicolumn{5}{|c|}{ Comitê de Ética em Pesquisa } \\
\hline & $\mathbf{A}$ & $\mathbf{B}$ & $\mathbf{C}$ & D & $\mathbf{E}$ \\
\hline \multicolumn{6}{|l|}{ Processo de distribuição dos projetos } \\
\hline Com base na expertise & 1 & - & - & 1 & - \\
\hline Disponibilidade para aprendizagem & 1 & - & - & - & - \\
\hline $\begin{array}{l}\text { Considera o volume que o relator já tem para } \\
\text { analisar }\end{array}$ & - & - & 1 & - & - \\
\hline Em reunião & - & - & - & - & 1 \\
\hline Quantidade aproximada de projetos analisados/mês & 150 & 10 & 15 & 15 & 15 \\
\hline \multicolumn{6}{|l|}{ Áreas temáticas dos projetos analisados pelo } \\
\hline \multicolumn{6}{|l|}{ Comitê de Ética em Pesquisa } \\
\hline Novos Equipamentos & - & - & 1 & - & - \\
\hline Insumos & - & - & 1 & - & - \\
\hline Dispositivos para saúde & - & - & 1 & - & - \\
\hline Novos Fármacos & - & - & 1 & - & - \\
\hline Reprodução Humana & - & - & 1 & - & - \\
\hline Outro & 1 & 1 & 1 & 1 & 1 \\
\hline \multirow{2}{*}{\multicolumn{6}{|c|}{$\begin{array}{l}\text { Áreas temáticas dos projetos analisados pelo } \\
\text { Comitê de Ética em Pesquisa/ mais frequência }\end{array}$}} \\
\hline & & & & & \\
\hline Novos Fármacos & - & - & 1 & - & - \\
\hline Outro & 1 & - & - & 1 & 1 \\
\hline Sem resposta & - & 1 & - & - & - \\
\hline Participação de consultor ad boc & 1 & Sem & Sem & Sem & Sem \\
\hline & & resposta & resposta & resposta & resposta \\
\hline \multicolumn{6}{|l|}{ Temáticas consultadas ao ad boc } \\
\hline Sistema de informação & 1 & - & - & - & - \\
\hline Antropologia & 1 & - & - & - & - \\
\hline Psicologia & 1 & - & - & - & - \\
\hline Teologia & 1 & - & - & - & - \\
\hline \multicolumn{6}{|l|}{$\begin{array}{l}\text { Periodicidade dos encontros para deliberar } \\
\text { sobre os projetos }\end{array}$} \\
\hline Quinzenalmente & 1 & - & 1 & - & - \\
\hline Mensalmente & - & 1 & - & 1 & 1 \\
\hline $\begin{array}{l}\text { Reuniões online/Projetos de relevância do Sistema } \\
\text { Único de Saúde }\end{array}$ & - & - & 1 & 1 & - \\
\hline \multicolumn{6}{|l|}{$\begin{array}{l}\text { Forma dos encontros para deliberar sobre os } \\
\text { projetos }\end{array}$} \\
\hline Presencial & - & - & 1 & 1 & - \\
\hline Videoconferência & 1 & 1 & 1 & 1 & 1 \\
\hline Sem resposta & - & - & - & - & - \\
\hline
\end{tabular}

Fonte: Elaboração própria.

Nota: Sinal convencional utilizado:

- Dado numérico igual a zero não resultante de arredondamento.

Quanto às outras áreas temáticas dos projetos analisados pelo CEP, foram descritas: CEP A, interdisciplinar (sem especificar quais); CEP B, estudos clínicos; CEP C, estudos qualitativos, área das ciências humanas, psicologia, nutrição, diagnósticos, avaliação de técnicas e procedimentos em saúde; CEP D, comportamento humano; e CEP E, alimentos, Saúde, Ciências Sociais e Humanas. Em relação às outras áreas temáticas dos projetos mais frequentemente analisados pelo CEP foram descritas, genericamente, como outras áreas: CEP A, ciências da educação, humanas e saúde; CEP B não respondeu; CEP C, estudos qualitativos, área das ciências humanas, 
psicologia, nutrição, diagnósticos, avaliação de técnicas e procedimentos em saúde; CEP D, comportamento humano; e CEP E, alimentos.

\section{Discussão}

Os dados sociodemográficos dos participantes do estudo apontam para maioria de membros de CEP do gênero masculino e formação em diferentes áreas do conhecimento, embora com predomínio da área de saúde, o que sugere a existência de composição não equilibrada dos membros. Tais dados corroboram estudos sobre a temática e que também apontaram existência de desequilíbrio na composição de CEP quanto ao gênero e à categoria profissional ${ }^{(1,13-14)}$.

Impende salientar que a distribuição balanceada de gênero, formação e categoria profissional na composição de CEP é uma exigência do CONEP para o registro desses comitês. A relevância de pessoas nas diferentes áreas de formação e categorias profissionais contribui para o aspecto de multidisciplinariedade e de multiprofissionalismo na composição dos CEPs $^{(8,15)}$.

Aliado a isso, a existência de composição com pessoas de áreas distintas pode favorecer discussões e análise mais aprofundadas dos projetos $^{(16-17)}$. Para compor esse aspecto, podem ser convidadas pessoas de diferentes instituições quando não houver profissionais da própria instituição ${ }^{(2)}$.

Por outro lado, ao tratar sobre a distribuição balanceada de gênero, o Manual Operacional para CEP, refere-se a homens e mulheres. De igual modo, a Norma Operacional (NOP) n. 001, de $2013^{(7)}$, ao definir sobre a composição do CEP, determina que deve haver pessoas dos dois sexos, isto é, não utiliza o termo gênero, mas mantém a ideia de homem e mulher ${ }^{(2,7)}$. Todavia, tem sido criticada a utilização do termo gênero como fator binário (feminino e masculino), pois a referência a binária de gênero não atende à concepção atual do tema ${ }^{(18-19)}$.

Os participantes deste estudo expressaram existência de atividades de treinamento inicial para ser membro do CEP e capacitação continuada, que também foram relatadas em outro estudo sobre $\operatorname{CEPs}^{(14)}$. As atividades para treinamento inicial e capacitação continuada são obrigatórias na operacionalização do CEP conforme determinação da $\operatorname{CONEP}^{(7)}$

As atividades de treinamento inicial são relevantes para que os novos membros possam ser capacitados quanto aos aspectos éticos e bioéticos e conheçam as normativas de pesquisas que envolvem seres humanos. Essas atividades devem incluir, entre outras, discussão acerca da função do CEP, apresentações de normas a respeito de pesquisas envolvendo seres humanos, indicação de referenciais teóricos sobre ética na pesquisa, leitura das resoluções sobre ética em pesquisa e do RI do respectivo $\mathrm{CEP}^{(2,20-21)}$.

Já a capacitação continuada pode ocorrer, entre outros meios, pela análise contínua dos protocolos de pesquisas, bem como por meio de cursos de extensão, graduação e pós-graduação $^{(14)}$. Tanto o treinamento inicial quanto a capacitação inicial possibilitam melhor percepção e compreensão dos protocolos de pesquisa discutidos e, consequentemente, que a apreciação ética seja realizada de forma ágil e adequada $^{(17)}$. Portanto, o treinamento inicial e a capacitação continuada, como partes integrantes da função educativa do CEP, ratificam a educação como meio capaz de preparar pessoas para a tomada de decisões de forma crítica e a adequada aplicação dos valores éticos ${ }^{(22)}$.

Ao serem questionados sobre incentivos fornecidos pelos CEPs para atuarem como membros, a maioria dos participantes deste estudo expressaram não haver tais incentivos. Todavia, a orientação da CONEP é que a instituição na qual o CEP encontra-se implantado desenvolva meios para haja estímulo e reconhecimento dessa atuação, como é o caso de carga horária específica, pontuação para avaliar produtividade acadêmica e progressão funcional. Além disso, uma vez comprovadas as despesas realizadas pelos membros de CEP para atividades no CEP, os valores gastos devem ser ressarcidos ${ }^{(2)}$.

Desse modo, torna-se relevante que haja auxílio financeiro, recursos materiais e humanos do local em que cada CEP esteja implantado e 
que seus membros sejam incentivados, como forma de valorização e reconhecimento do trabalho voluntário dessas pessoas. Por essa razão, a contabilização das horas de atividades no CEP para efeito de trabalho efetivo na instituição, o reconhecimento institucional e curricular do trabalho de seus membros como pontuação para progressão funcional são apontadas como possíveis alternativas para mitigar as limitações desse sistema e estimular a participação dos membros de $\operatorname{CEP}^{(23)}$.

As informações no presente estudo sobre o tempo de implantação dos CEPs demonstram que esses Comitês possuem longa experiência na apreciação de projetos envolvendo pesquisas com seres humanos, mas há um contraste quando se verifica que os membros permanecem no cargo entre dois e três anos, com direito somente a uma recondução ao cargo. Similarmente, estudo que analisa as características dos CEP distribuídos no território brasileiro aponta para o curto período de permanência dos membros, o que revela alta rotatividade no cargo e pouca experiência. Todavia, a experiência em pesquisa é essencial para o fortalecimento do Sistema CEP/CONEP e para que os membros dos CEPs realizem a revisão ética dos protocolos de forma adequada, o que, entre outros requisitos, exige tempo e experiência ${ }^{(17)}$.

A experiência dos membros de CEPs ocorre à proporção em que estes são reconduzidos ao cargo, o que promove aquisição de novos conhecimentos e aprimoramento da experiência por meio da contínua análise ética das pesquisas. Desse modo, a renovação do mandato possibilita a permanência de membros experientes no CEP, e, consequentemente, favorece o aprofundamento das discussões e a capacitação de novos membros quanto aos conhecimentos necessários para a avaliação dos projetos.

Os CEPs deste estudo mantêm relacionamento com o público/pesquisadores, com outros CEPs e com a CONEP, com destaque para a página eletrônica. Estudo ${ }^{(24)}$ aponta a importância desta relação na medida que proporciona proteção da liberdade e do bem-estar dos participantes e a promoção da confiança pública da pesquisa. Especialmente em relação aos CEPs, por meio desse relacionamento é possível haver trocas de experiências e informações para subsidiar a solução de casos semelhantes. Aliado a isso, as trocas de experiências, assim como as atividades para capacitação inicial e capacitação continuada, estão em consonância com a função educativa do CEP, para possibilitar que seus membros desenvolvam a capacidade de reflexão e tomem decisões éticas, tendo em vista a defesa e a proteção dos participantes de pesquisa ${ }^{(17,25)}$.

A existência de diferentes formas de distribuição dos protocolos de pesquisa entre os membros dos CEPs vista neste estudo, entre os quais, as áreas de atuação e de formação de cada um de seus membros, a expertise, o perfil e a disponibilidade do membro para aprender, contribui para estimular a aprendizagem dos membros e possibilitar a sua inserção ao estudo das diferentes temáticas dos protocolos de pesquisa submetidos para apreciação. Caso haja dificuldade em analisar um protocolo de pesquisa em particular, esta poderá ser resolvida pela participação do membro ad hoc especialista no tema em discussão.

Essa diversificação também foi vista em estudo $^{(26)}$ realizado em Minas Gerais a respeito da experiência de CEP, o qual ressalta a análise de projetos de pesquisa nas diversas áreas do $\mathrm{CO}^{-}$ nhecimento com destaque para as ciências da saúde. Frise-se que essa diversidade de projetos de pesquisa reforça a necessidade de se pensar na distribuição dos projetos para, não somente se oportunizar o esquema de rodízio sugerido pela CONEP, mas possibilitar que os conhecimentos dos membros sejam ampliados, uma vez que o fomento à aprendizagem também deve fazer parte do papel educativo de cada CEP.

Entre as limitações do estudo, cita-se a baixa adesão dos membros coordenadores de CEP. Contudo, este estudo tem relevância científica ao conseguir caracterizar os profissionais que compõem os Comitês em seu processo de trabalho e, sobretudo, apresenta para a comunidade acadêmica os desafios organizacionais, institucionais e estruturais dos comitês de ética 
em pesquisa com base na amostragem de um município baiano de grande porte.

\section{Conclusão}

A pesquisa possibilitou caracterizar os CEPs do estudo com destaque para o fato de que todos possuem atuação há mais de oito anos e uma recondução de mandato, o que demonstra experiência consolidada em pesquisa. Desse modo, a recondução do mandato possibilita que os membros mais experientes se mantenham no CEP contribuindo para o aprofundamento das discussões durante a apreciação dos protocolos de pesquisas, bem como para capacitação de novos membros. Ademais, essa experiência representa um ganho essencial para que o sistema CEP/CONEP seja fortalecido nos processos de apreciação ética.

As contribuições deste estudo englobam os campos acadêmicos e os profissionais que se dedicam à pesquisa envolvendo seres humanos. É importante que se conheça os CEPs, sua composição, seu papel e como atuam na apreciação de projetos de pesquisa pela relevância que estes comitês têm quanto ao controle social das pesquisas, bem como para subsidiar políticas públicas voltadas para o fortalecimento do Sistema CEP/CONEP, para que seus membros continuem atuando em prol da proteção dos direitos dos participantes da pesquisa. Portanto, é necessário que se fomente para a comunidade acadêmica a existência e atuação dos CEPs em disciplinas específicas, bem como pelas leituras de textos científicos. Do mesmo modo, na seara profissional, é relevante fomentar publicações relacionadas aos CEPs como forma de ratificar os projetos de pesquisas apreciados por órgãos comprometidos na promoção de apreciação ética dos projetos, tendo em vista proteção integral dos participantes da pesquisa e proteção do pesquisador ao validar projetos de pesquisa como proposta ética apropriada.

\section{Colaborações:}

1 - concepção, projeto, análise e interpretação dos dados: Rosinete Souza Barata, Karla
Ferraz dos Anjos, Alciene Pereira da Silva e Darci de Oliveira Santa Rosa;

2 - redação do artigo e revisão crítica relevante do conteúdo intelectual: Rosinete Souza Barata, Karla Ferraz dos Anjos, Alciene Pereira da Silva, Julia Barbosa de Magalhães e Darci de Oliveira Santa Rosa;

3 - aprovação final da versão a ser publicada: Rosinete Souza Barata, Karla Ferraz dos Anjos, Alciene Pereira da Silva, Julia Barbosa de Magalhães e Darci de Oliveira Santa Rosa.

\section{Referências}

1. Jácome MQD, Araujo TCCF, Garrafa V. Comitês de ética em pesquisa no Brasil: estudo com coordenadores. Rev Bioét. 2017;25(1):61-71. DOI: 10.1590/1983-80422017251167

2. Brasil. Ministério da Saúde. Conselho Nacional de Saúde. Comissão Nacional de Ética em Pesquisa. Manual operacional para comitês de ética em pesquisa [Internet]. Brasília (DF); 2007. (Série A. Normas e Manuais Técnicos) (Série CNS Cadernos Técnicos) [cited 2021 Jul 4]. Available from: https://conselho.saude.gov.br/biblioteca/ livros/Manual_Operacional_miolo.pdf

3. Passini R, Pinheiro M, Montagner M. Declaração de Helsinque: flexibilização do uso do placebo, um interesse do mercado farmacêutico. Rev Bras Bioét. 2018;14:1-20. DOI: https://doi.org/10.26512/ rbb.v14i0.14687

4. Ssali A, Poland F, Seeley J. Experiences of research ethics committee members and scientists of the research protocol review process in Uganda: a case study. Int Health. 2020;12(6):541-2. DOI: https://doi.org/10.1093/ inthealth/ihaa047

5. Brasil. Ministério da Saúde. Conselho Nacional de Saúde. Resolução no 466, de 12 de dezembro de 2012. Aprova as diretrizes e normas regulamentadoras de pesquisas envolvendo seres humanos [Internet]. Brasília (DF); 2012 [cited 2021 Jul 4]. Available from: http://conselho.saude. gov.br/resolucoes/2012/Reso466.pdf

6. Batista KT, Seidl EMF. Sistema brasileiro de revisão ética em pesquisa: percepção de pesquisadores. Rev Bras Bioét. 2018;14(e15):1-18. DOI: https://doi.org/10.26512/rbb.v14i0.20621

7. Brasil. Ministério da Saúde. Conselho Nacional de Saúde. Comissão Nacional de Ética em 
Pesquisa. Norma Operacional nº 001, de 2013. Dispõe sobre a organização e funcionamento do Sistema CEP/Conep e procedimentos para submissão, avaliação e acompanhamento do desenvolvimento da pesquisa envolvendo seres humanos no Brasil [Internet]. Brasília (DF); 2013 [cited 2021 Jul 4]. Available from: https:// conselho.saude.gov.br/images/comissoes/conep/ documentos/normas-resolucoes/norma_operacional_ n_001-2013_procedimento_submisso_de_projeto. pdf

8. Brasil. Ministério da Saúde. Conselho Nacional de Saúde. Comissão Nacional de Ética em Pesquisa. Orientações para Registro de Comitê de Ética em Pesquisa (CEP) [Internet]. Brasília (DF); 2019 [cited 2021 Jan 1]. Available from: https:// conselho.saude.gov.br/Web_comissoes/conep/ aquivos/cep/orientacoes_gerais/Orientacoes_ para_Registro2019.pdf

9. Brasil. Ministério da Saúde. Secretaria de Ciência, Tecnologia e Insumos Estratégicos. Departamento de Ciência e Tecnologia. Agenda nacional de prioridades de pesquisa em saúde [Internet]. Brasília (DF); 2015 [cited 2021 Jul 4]. Available from: http://brasil.evipnet.org/wp-content/uploads/ 2017/07/ANPPS.pdf

10. Ma X, Wang Y, Gao T, He Q, He Y, Yue R, et al. Challenges and strategies to research ethics in conducting COVID-19 research. J Evid Based Med. 2020;13(2):173-7. DOI: 10.1111/jebm.12388

11. Mathur R, Thakur K, Hazam RK. Highlights of Indian Council of Medical Research National Ethical Guidelines for Biomedical and Health Research Involving Human Participants. Indian J Pharmacol. 2019;51(3):214-21. DOI: 10.4103/ 0253-7613.262456

12. Brasil. Ministério da Saúde. Conselho Nacional de Saúde. Comissão Nacional de Ética em Pesquisa. Mapa dos CEPs [Internet]. Brasília (DF); 2019. Atualizada em março de 2020 [cited 2021 Jul 4]. Available from: https://plataformabrasil. saude.gov.br/visao/centralSuporteNova/consultar ComiteEtica/consultarComiteEtica.jsf

13. Contijo PAM. Capacitação de membros dos comitês de ética em pesquisa no município de Belo Horizonte, Minas Gerais através do E-Learning TRREE [tese]. Belo Horizonte (MG): Faculdade de Medicina, Universidade Federal de Minas Gerais; 2017.

14. Barbosa IPR, Francisco FR, Martinez LL. Perfil dos Comitês de Ética em Pesquisa no Brasil: uma análise do sistema de regulação ética da pesquisa clínica, pontos estratégicos e desafios. Arq Med Hosp Fac Cienc Med Santa Casa São Paulo. 2018;63(3):145-59. DOI: https://doi.org/ 10.26432/1809-3019.2018.63.3.145

15. Hunt A, Saenz C, Littler K. The global forum on bioethics in research meeting, "ethics of alternative clinical trial designs and methods in low- and middle-income country research": emerging themes and outputs. Trials. 2019;20(Suppl 2). DOI: https://doi.org/10.1186/s13063-019-3840-3

16. González-Duarte A, Zambrano-González E, Medina-Franco H, Alberú-Gómez J, DurandCarbajal M, Hinojosa CA, et al. II. The Research Ethics Involving Vulnerable Groups. Rev Invest Clin. 2019;71(4):217-25. DOI: https://doi. org/10.24875/RIC. 19002812

17. Jácome MQD, Araújo TCCF. Concepções de membros de comitês de ética em pesquisa acerca do processo de avaliação. Amazôn Rev Antropol. 2018;10(2):392-411. DOI: 10.18542/amazonica. v10i2.6508

18. Heidari S, Babor TF, Castro P, Tort S, Curno M. Equidade de sexo e gênero na pesquisa: fundamentação das diretrizes SAGER e uso recomendado. Epidemiol Serv Saúde. 2017;26(3):665-76. DOI: https://doi.org/10.5123/ s1679-49742017000300025

19. Darsie C, Saraiva ES. Gênero e diversidade sexual na educação. Reflex Ação. 2016;24(1):1-6. DOI: https://doi.org/10.17058/rea.v24i1.7495

20. Pasic MB, Vidrih B, Sarac H, Pasic H, Vujević L, Koruga AS, et al. Clinical trials in developing countries - ethical considerations. Psychiatr Danub. 2018;30(3):285-91. DOI: 10. 24869/psyd.2018.285

21. Salas SP. Experiencia en el Foro Global de Bioética en Investigación. Desafíos para la revisión ética en Chile. Rev Med Chil. 2018;146(5):653-9. DOI: 10.4067/s0034-98872018 000500653

22. Walker A, Boyce A, Duggal P, Thio CL, Geller G. Genomics and Infectious Diseases: Expert Perspectives on Public Health Considerations regarding Actionability and Privacy. Ethics Hum Res. 2020;42(3):30-40. DOI: https://doi.org/10.1002/eahr.500051

23. Barbosa AS, Corrales CM, Silbermann M. Controvérsias sobre a revisão ética de pesquisas em ciências humanas e sociais pelo Sistema 
CEP/Conep. Rev Bioét. 2014;22(3):482-92. DOI: https://doi.org/10.1590/1983-80422014223031

24. Goldstein CE, Weijer C, Brehaut JC, Fergusson DA, Grimshaw JM, Horn AR, et al. Ethical issues in pragmatic randomized controlled trials: a review of the recent literature identifies gaps in ethical argumentation. BMC Med Ethics. 2018;19(14). DOI: https://doi.org/10.1186/s12910018-0253-x

25. Aarons D. Ressearch in epidemic and emergency situations: a model for collaboration and expediting ethics review in two Caribbean countries. Dev World Bioeth. 2018 Dec;18(4): 375-84. DOI: https://doi.org/10.1111/dewb.12157

26. Paiva PA, Costa SM, Dias OV, Lopes VSVBV, Souto DGB, Silva DL. The experience of the research ethics committee at a public university in the state of Minas Gerais, Brazil. Rev bioét. 2015;23(1):169-77. DOI: https://doi. org/10.1590/1983-80422015231057

Recebido: 6 de julho de 2021 Aprovado: 15 de setembro de 2021

Publicado: 5 de outubro de 2021

A Revista Baiana de Enfermagem utiliza a Licença Creative Commons - Atribuição-NãoComercial 4.0 Internacional. https://creativecommons.org/licenses/by-nc/4.0/

Este artigo é de acesso aberto distribuído sob os termos da Licença Creative Commons (CC BY-NC).

Esta licença permite que outros remixem, adaptem e criem a partir do seu trabalho para fins não comerciais. Embora os novos trabalhos tenham de lhe atribuir o devido crédito e não possam ser usados para fins comerciais, os usuários não têm de licenciar esses trabalhos derivados sob os mesmos termos. 